\title{
DEBATES
}

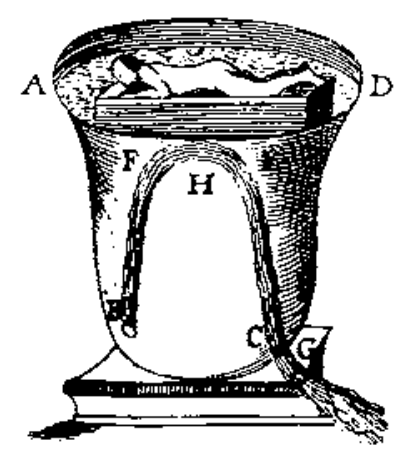

\section{SISTEMAS DE REPRESENTACIÓN Y APRENDIZAJE DE ESTRUCTURAS NUMÉRICAS}

\author{
CASTRO MARTíNEZ, E., RICO ROMERO, L. y ROMERO ALBALADEJO, I. \\ Departamento de Didáctica de la Matemática. Universidad de Granada. España.
}

Iniciamos esta sección de debates recogiendo los tex tos correspondientes al foro de investigación sobre el tema «NonElementary Numerical Thinking» deI $20^{\circ}$ congreso deI International Group for the Psychology of Mathematics Education, celebrado en Valencia en julio de 1996.

Los foros de investigación de los congresos de PME están concebiđos para generar un debate alređedor de un tema de investigación sobre el que se haya producido ya un trabajo prolongado por parte de uno o varios grupos de investigación. En este caso, los miembros del grupo de investigación «Pensamiento numérico" de la Universidad de Granada, Luis Rico, Encarna Castro e Isabel Romero presentaron el trabajo The Role of Representation Systems in the Learning of Numerical Structures, que, previamente a Ia celebración del congreso, se envió a Alan Bell y Richard Lesh para que elaboraran sendas rêplicas.

En las actas del congreso están publicados el original inglés del trabajo đe Rico, Castro y Romero y una versión de tan sólo nueve páginas de la réplica de Richard Lesh, también en inglés (Puig, L. y Gutiérrez, A., eds. Proceedings of the 20 th Conference of the International Group for the Psychology of Mathematics Education, vol. 1, pp. 87-102 y addenda, pp. 33-11, respectivamente). Lo que presentamos aquí no sólo hace más accesible este debate a los lectores de Iengua castellana, sino que da cuenta de forma más completa de lo que se prođujo en el congreso, ya que, además de la traducción castellana del trabajo đe Rico, Castro y Romero, publicamos una versión mucho más larga de la réplica de Lesh y un texto escrito especialmente para esta ocasión por Alan Bell a partir de Io que fue su réplica oral en el congreso. 


\section{INTRODUCCIÓN}

Este trabajo ha sido presentado para su discusión y debate en la sección Research Forum del XX encuentro del International Group for the Psychology of Mathematics Education, celcbrado en Valencia en 1996. En el mismo nos ocupamos del aprendizaje de conceptos numéricos en el sistema cscolar y de la utilidad que tiene la noción de representación para analizar e interpretar la comprensión de tales conceptos por parte de escolares de 12-15 años. Nuestro grupo de investigación está interesado en el estudio de las dificultades que los jóvenes encuentran en el mancjo de las estructuras numéricas cuando se enfrentan a nociones de matemática avanzada. Presentamos aquí los objetivos generales y algunos resultados de uno de los trabajos de investigación realizados por nuestro grupo, que comparten este planteamiento.

El instrumento elegido para poner de manifiesto algunas carencias del currículo y para observar e interpretar el trabajo de los alumnos en su aprendizaje de conceptos numéricos $\mathrm{y}$, por consiguiente, en la construcción y desarrollo de su pensamiento numérico, es la noción de representación, noción cuyo interés y utilidad para la investigación en educación matemática es conocido y ha sido objetu de reflexión continuada (Janvier, 1978, 1987; Kaput, 1987, 1992; Goldin, 1993; Duval, 1993, 1995). Aunque muchas de las consideraciones que realizamos son válidas para otros campos de las matcmáticas, nuestra aportación en este trabajo se limita explícitamente a conceptos y estructuras numéricas.

Dado que to hay un significado unívoco para el concepto de representación, consideramos neccsario precisat el sentido en que vamos a utilizar este término, lo cual vamos a hacer en tres delimitaciones sucesivas que preceden a la presentación de los resultados de la investigación mencionada.

\section{SOBRE LA NOCXÓN DE REPRESENTACIÓN}

\section{Caracterización general}

La historia de la filosofía y de la ciencia muestra la riqueza de sentidos e interpretaciones que tiene este concepto (Ferrater, 1981), algunos de los cuales son importantes para las actuales líneas de investigación en educación matemática (Goldin, 1993).

Un primer punto de interés para nosotros está en lá idea de que una representación es siempre represen. tación de algo. El concepto de representación «da por supuesta la consideración de dos entidades relacionadas, pero funcionalmente separadas". Uno de estos entes se denomina cl objeto representante (o representación), el otro es el objeto representado. También hay implícita cierta correspondencia entre el mundo de Jos objetos representantes y el mundo de los objetos representados.
De esta manera, «cualquier especificación particular de la noción de representación debería describir, al menos, cinco entidades»:

1) los objetos representados;

2) los objetos representantes;

3) qué aspectos del mundo representado se representan;

4) qué aspectos del mundo representante realizan la representación;

5) la correspondencia entre ambos mundos o conjuntos.

«En buena parte de los casos importantes uno o ambos de los mundos pueden ser entidades hipotéticas e incluso abstracciones» (Kaput, 1987).

Por lo que a nuestro estudio se refiere, consideramos necesario distinguir entre los conceptos y estructuras numéricas y los sistemas de representación mediante los que se expresan tales conceptos y estructuras. Cuando identificamos los números naturales con los numerales, que se obtienen mediante las reglas de escritura de números del sistema decimal de numeración, olvidamos que la escritura decimal es sólo una forma de representar números, enunciados y demostraciones numéricas mediante combinación lineal de las sucesivas potencias de 10. El teorema fundamental de la aritmética propone una representación diferente de cada número como producto de factores primos, que muestra su estructura multiplicativa.

Aunque no es usual, nosotros consideraremos cuáles son los aspectos y propiedades de los números naturales que se destacan mediante cada tipo de simbolización. Cada uno de los modos de representación de los números naturales, junto con las reglas que los acompañan, propone una caracterización distinta del concepto de número natural. Identificar los números con una cualquiera de sus notaciones es una simplificación escolar, inadecuada para la investigación en educación matemática. Por ello diferenciamos entre los números y sus representaciones

Una segunda idea importante para nosotros es cl uso, en la filosofía contemporánea, del término representación para referirse a cualquier cosa que puede evaluarse semánticamente (Dancing y Sosa, 1993). De las representaciones puede decirse que: son verdaderas; se refieren a; son verdaderas de algo; son acerca de algo; son precisas; etc. Contenido es el término técnico utilizado para denominar aquello que en una representación la hace semánticamente evaluable. Así, de un enunciado, se dice que tiene como contenido una proposición o conđición de verdad; de un término, se dice que tiene un concepto como contenido; de una gráfica, que expresa una relación adecuada entre sus componentes. Desde este planteamiento son representaciones las expresiones simbólicas, los enunciados, Ios diagramas, los gráficos y otras notaciones usuales de las matemáticas. Estos contenidos son objeto de estudio en matemáticas. 


\section{Estructuras numéricas y sistemas de representación}

La moderna conceptualización de los números está basada en la noción de sistema; hablando con cierta precisión no nos referiremos a conceptos numéricos simplemente, sino a sistemas o estructuras numéricas. Una estructura numérica consiste en un conjunto de entes abstractos expresados simbólicamente, dotado de unas operaciones o modos de componer esos números y de unas relaciones, mediante las que se comparan dichos entes. La consideración conjunta de los entes, sus operaciones y sus relaciones es lo que caracteriza una estructura numérica (Feferman, 1989). La estructura de cada sistema viene determinada por un grupo reducido de grandes y potentes ideas (Fey, 1990).

Los matemáticos trabajan con símbolos y representaciones significantes (Kaput, 1987), cuya naturaleza y modo de empleo han ocupado la atención de investigadores y pensadores matemáticos a lo largo de la historia de esta ciencia.

El conjunto de signos, símbolos y reglas para expresar o representar una estructura matemática ha de responder a su carácter sistémico, por ello se habla de sistemas matemáticos de signos (Kieran y Filloy, 1989), sistemas de notación (Kaput, 1992) o sistemas semióticos (Duval, 1993). Nosotros, en este trabajo, utilizaremos el término sistemas de representación para referimos a los modos de expresar y simbolizar determinadas estructuras numéricas, mediante unos signos, unas reglas y unos enunciados; el sistema decimal de numeración o notación decimal constituye un ejemplo paradigmático de sistema de representación familiar para los números naturales.

La consideración estructural de los números y la elección que hemos hecho de distinguir entre los números y sus representaciones nos lleva a la fundamentación formalista de las matemáticas. En los planteamientos formales de Peano y Hilbert, los campos numéricos se caracterizan como campos operatorios (Badiou, 1990). El formalismo destaca la consideración técnica de los números, como herramientas con las que llevar a cabo determinados procesos, mediante el cumplimiento de unas reglas, y la posibilidad de establecer una multiplicidad de relaciones entre los diferentes números. Signos y símbolos, junto con la sintaxis mediante la que se combinan para dar paso a expresiones más complejas y formulas bien hechas, juegan un papel central dentro de la escuela formalista, complementados por los procedimientos finitistas para probar los enunciados y fórmulas de cada sistema numérico (Von Neumann, 1964).

Por otra parte, en su base epistemológica, los conceptos matemáticos, al constituirse a partir de relaciones entre objetos, fenómenos o conceptos previos, y no referirse a los propios objetos o fenómenos físicos, se consideran entidades abstractas cuya ex presión viene dada por enunciados y demostraciones que necesitan de algún sistema simbótico; es decir, los conceptos vienen dados mediante una o varias representaciones específicas. Se consideran aquí dos niveles de representación: los hechos o conceptos concretos (p.e., la unidad) representados por símbolos específicos (p.e., 1), y las relaciones entre conceptos (p.e., uno y uno hacen dos) representadas por enunciados simbólicos (p.e., 1 + I = 2) (Körner, 1977). Asumimos una base fenomenologica para conceptos y relaciones numéricas.

Además, no hay sistema simbólico que agote en su totalidad la complejidad que encierra cada concepto matemático; es por ello que cada concepto matemático đispone de más de un sistema de representación. A su vez, cada uno de estos sistemas destaca y pone de manifiesto algunas propiedades importantes del concepto, a la par que oculta o dificulta la expresión de otras propiedades. Admitimos como sistemas de representación en matemáticas: el lenguaje natural, las figuras y gráficas, las diferentes escrituras simbólicas, las tablas y cuadros y las notaciones algorítmicas que se expresan por un modo de operar.

\section{Representación y cognición}

Desde el interés de la educación matemática hemos de considerar los conceptos matemáticos conectados con la actividad mental de las personas. Siguiendo a Wittgenstein (1988), cuando éste reflexiona sobre los «diversos juegos de lenguaje matemáticos» y entre ellos el concepto de número (op. cit., pp. 65-68), sostenemos que cada concepto matemático viene establecido por sus diferentes significados y usos y, por tanto, por sus representaciones. Son los usos de cada concepto los que establecen por extensión su campo semántico, y cada modo significativamente distinto de entender un concepto necesita de un sistema de simbolización propio, de algún modo de representación para ser distinguible. Abocamos así en la distinción entre representaciones internas y externas. Las representaciones internas u objetos del pensamiento, ubicadas en las mentes individuales de los sujetos, son distintas de las representaciones externas, de carácter semiótico, dadas por signos, símbolos o gráficos, de las que hemos hablado en el apartado anterior.

El uso de la noción de representación para caracterizar Ios estados mentales y las actividades de los sujetos constituye un dato destacable en el desarrollo reciente de la psicología cognitiva (Guttenplan, 1994). Asumimos que los procesos cognitivos son aquellos procesos que manipulan representaciones. Una diferencia esencial entre los procesos cognitivos y los que no lo son es, precisamente, que los primeros se pueden valorar epistémicamente. Dado que ślo algo con contenido puede evaluarse desde el punto de vista epistémico, únicamente pueden considerarse como cognitivos los procesos en tanto que implican representaciones. En el desarrolto de los procesos, que implican pensamiento numérico por parte de los sujetos, resulta esencial un dominio mental adecuado de las representaciones externas, que potencia la comprensión de los conceptos numéricos.

Siguiendo a Wittrock (1990), consideramos que la comprensión es «una representación estructural o conceptualmente ordenada de las relaciones entre las partes de la información que se debe aprender, y entre esa infor- 
mación y esas ideas y nuestra base de conocimientos y experiencia». Los distintos sujetos presentan comprensiones diferentes sobre un mismo concepto o estructura matemática debido a que sus representaciones mentales tienen contenidos diferentes. La relación entre las representaciones internas y las externas es clave en el estudio de los fenómenos de comprensión.

\section{Balance}

La noción de representación en educación matemática debe tener en cuenta la dualidad del concepto: «Para pensar sobre ideas matemáticas y comunicarlas necesitamos representarlas de algún modo. La comunicación requiere que las representaciones sean externas, tomando Ia forma de lenguaje oral, símbolos escritos, dibujos u objetos físicos. [...] Para pensar sobre ideas matemáticas necesitamos representarlas internamente, de manera que permita a la mente operar sobre ellas» (Hiebert y Carpenter, 1992). El conocimiento matemático es accesible solamente a partir de las representaciones externas, que son los datos para este conocimiento. El fenómeno de la representación también concierne al funcionamiento mismo del pensamiento, ocupa una posición central en el aprendizaje de las matemáticas.

Esta dualidad de la noción đe representación le convierte en un instrumento adecuado para el estudio de los fenómenos de comprensión. También es útil para los intereses del investigador cuando se propone dar cuenta de los modos en que los sujetos procesan una estructura numérica.

Somos conscientes de algunos equívocos que pueden surgir con la denominación sistemas de representación, puestos de manifiesto por Kaput (1992) en el sentido de que, al tomar el término sistema de representación (representantes) como básico, hay que caracterizar el concepto de número (representado) independientemente. No obstante, entendemos que esta dificultad se mantiene igualmente si en vez de hablar de representaciones hablamos de notaciones o simbolizaciones, ya que, a su vez, los símbolos deben expresar o notar un concepto cuya caracterización deberá hacerse independientemente de tales notaciones. Por elio hemos desarrollado con cierta extensión algunas ideas que fundamentan nuestra posición.

La complejidad considerada ha permitido destacar las dimensiones fenomenológica y cognitiva del pensamiento numérico. También ha posibilitado tomar distancias del planteamiento platónico que postula la existencia de los conceptos matemáticos independientemente de condicionantes espaciotemporales y de la actividad mental de lo sujetos (Kitcher, 1984).

\section{DELIMITACIÓN DEL TRABAJO}

\section{Antecedentes}

A comienzos de los ochenta hay dos campos conceptuales cuyo estudio sostenido se inicia sobre la base de la nocion de representación.
Uno de estos campos es el relativo al concepto de función; en los estudios realizados destacan los diversos sistemas de representación para las funciones y se detectan algunas dificultades de comprensión sobre este concepto, debidas a problemas de traducción entre dichos sistemas. Los estudios de Janvier, que culminan en su tesis en 1978, están entre los más conocidos, dando lugar posteriormente a los materiales elaborados por el Shell Centre de la Universidad de Nottigham, en los que se aborda una enseñanza por diagnóstico sobre este campo conceptual basada en las representaciones gráficas.

El segundo de los campos de estudio trabaja sobre el concepto de número racional, sobre la base de considerar y analizar diferentes sistemas de representación para este campo numérico. Los trabajos de Behr, Lesh, Post y Silver (1983) se encuentran entre los pioneros en el estudio de este conjunto numérico, que aún continúa ofreciendo resultados productivos.

En 1984 se celebra un simposio en la Universidad de Quebec en Montreal, organizado por el CiRADE, para presentar y discutir las últimas etapas de un proyecto de investigación sobre representación. Resultado de este simposio es el libro Problems of Representation in the Teaching and Learning of Mathematics (1987), en el que se plantea el estado de la cuestión sobre la potencialidad para la investigación en educación matemática del concepto en estudio.

El interés del tópico se ha puesto de manifiesto por la existencia del Working Group on Representations, en el seno del International Group for the Psychology of Mathematics Education, hasta el año 1995. Goldin, que fue coordinador de este grupo de trabajo, pone de manifesto el interés del tópico en cuestión: «Las representaciones son constructos teóricos claves en la psicología de la educación matemática. El significado de este término es bastante amplio e incluye:

a) ejemplificaciones físicas externas (incluyendo entornos computacionales): una situación física externa estructurada o un conjunto de situaciones que pueden describirse matemáticamente o verse como encarnación de una idea matemática;

b) explicitaciones lingüísticas: aspectos verbales, sintácticos o semánticos del lenguaje mediante los que se proponen problemas y se discuten las matemáticas;

c) constructos matemáticos formales: supone un significado diferente de representación que enfatiza aún sobre un problema del entorno externo al individuo; se trata de un análisis formal, estructural o matemático de una situación o un conjunto de situaciones;

d) representaciones cognitivas internas: «las representaciones individuales, internas, de los estudiantes para las ideas matemáticas, así como los sistemas de representación cognitiva en un sentido más amplio que puede describir los procesos del aprendizaje humano y la resoJución de problemas en matemáticas» (1993). 
Desde una aproximación semiótica, el profesor Duval de la Universidad de Estrasburgo, está trabajando sobre la noción de representación y la comprensión de los objetos matemáticos desde comienzos de la década de los ochenta; su trabajo Semiosis y noesis (1993) es una aportación valiosa en este sentido.

No hemos encontrado, sin embargo, antecedentes del estudio que aquí presentamos sobre la comprensión del término general de una sucesión, que se basa en el uso de diversos sistemas de representación para números.

\section{Objetivos y supuestos}

El objetivo central del trabajo que aquí presentamos es poner de manifiesto la pluralidad de sistemas de representación mediante los que se expresan las estructuras numéricas. Sostenemos que cada sistema numérico, como complejo de entes, relaciones y operaciones, no puede expresarse en su totalidad mediante un único sistema de representación. Es necesaria la actuación coordinada de varios sistemas de representación para poner de manifiesto aspectos esenciales de las estructuras numéricas convencionales. En particular, las representaciones gráficas son esenciales para la comprensión de las estructuras numéricas.

Nos proponemos ejemplificar estas ideas mediante una investigación llevada a cabo por nosotros: «Exploración de patrones numéricos mediante configuraciones puntuales» (Castro, 1994), que estudia la integración de tres sistemas de representación para los números naturales con el fin de profundizar sobre la comprensión -por escolares de 12-14 años, de conceptos y procedimientos implicados en la noción de término general de una sucesión de números naturales.

\section{MARCO DE INVESTIGACIÓN}

\section{EI grupo de investigación Pensamiento Numérico}

El trabajo que aquí presentamos se enmarca en la línea de trabajo del grupo de investigación Pensamiento Numérico. Este grupo desarrolla una línea de indagación y estudio dentro de la educación matemática sobre los fenómenos de enseñanza, aprendizaje y utilización de conceptos numéricos, tanto en el medio escolar como social. El campo general en el que se desenvuelve la investigación comprende el estudio de los diferentes sistemas cognitivos y culturales con que los seres humanos asignan y comparten significado utilizando diferentes estructuras numéricas.

Desde una perspectiva amplia, el marco conceptual en el que se sitúa Pensamiento Numérico tiene unas bases diversificadas:

1) Asume que la construcción del conocimiento matemático es un fenómeno social y cultural cuya importancia para la sociedad tecnológica actual es determinante. Tiene en cuenta que la educación matemática desempe- ña un papel relevante en la transmisión de los significados y valores compartidos en nuestra sociedad. La educación matemática debe considerar críticamente el conocimicnto matemático y las acciones comunicativas mediante las que se transmite.

2) Considera como objeto de refjexión el campo de las matemáticas, que comienza en la aritmética escolar y las nociones básicas de número, avanza por los sistemas númericos superiores (enteros, racionales y decimales) y continúa con el estudio sistemático de las relaciones numéricas, el cual aborda la teoría de números, la iniciación a los procesos infinitos que dan lugar al sistema de los números reales y los principales conceptos del análisis, vistos desde una perspectiva numérica. Denominamos conocimiento numérico a este modo de priorizar y caracterizar determinadas ramas de la matemática mediante uso de las herramientas conceptuales que llama. mos estructuras numéricas.

3) Tienc una orientación esencialmente curricular, entendiendo que la orientación de la investigación en educación matemática debe resolver los problemas de la práctica escolar, considerando el carácter sistémico de cualquier plan de formación en matemáticas dentro del sistema educativo. La valoración del currículo como un plan operativo con diferentes niveles de reflexión e implementación es uno de los rasgos definitorios de nuestra línea. La preocupación por los problemas que aparecen al considerar la evaluación escolar en matemáticas merecen una especial consideración.

4) EI estudio de los errores y dificultades en la comprensión de los escolares sobre los campos conceptuales antes mencionados constituye, junto con el conocimiento de la organización, sistematización y desarrollo de diferentes competencias cognitivas que encuentran un modo de actuación en el marco de una estructura numérica, la orientación psicológica de nuestras investigaciones.

5) Finalmente, está comprometido en la formación inicial y permanente del profesorado de matemáticas y considera objetivo prioritario el aumento de la autonomía intelectual y profesional del educador matemático.

Los tópicos principales trabajados por el grupo son:

- Cognición numérica.

- Bases filosóficas y epistemológicas del concepto de número.

- Desarrollo histórico de los sistemas numéricos.

- Aritmética escolar.

- Sistemas numéricos superiores.

- Relaciones numéricas y secuencias de números.

- Procesos infinitos.

Más concretamente, los objetivos del grupo se han centrado en el estudio de:

a) la organización conceptual de sistemas simbólicos de codificación, válidos para la expresión y comunicación de los conceptos y relaciones de una estructura numérica y las interrelaciones entre tales sistemas; 
Esquema 1

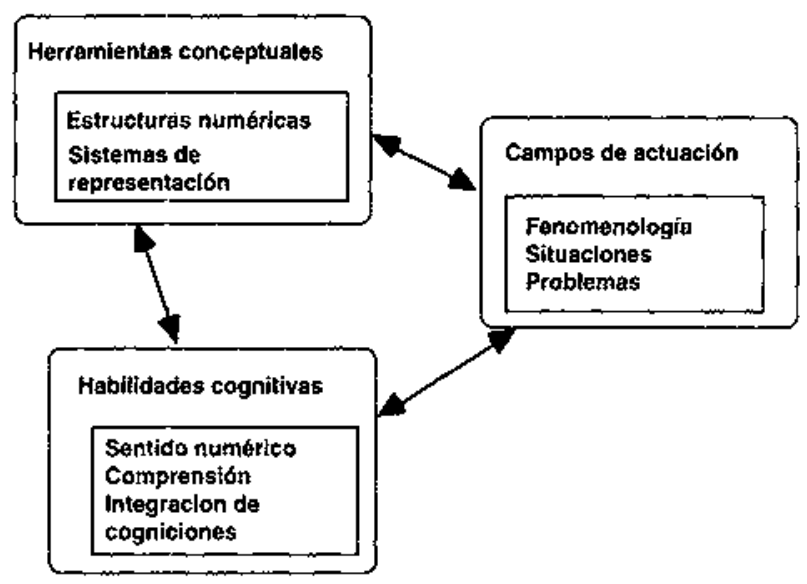

b) la elaboración y construcción mental de los sistemas simbólicos mencionados, así como la organización, sistematización y desarrollo de diferentes competencias cognitivas basadas en las estructuras numéricas;

c) Ios modos de abordar, interpretar y, en su caso, responder a una variedad de fenomenos cuestiones y problemas que admiten ser analizados mediante conceptos y procedimientos que forman parte de una estructura numérica.

El modelo que proponemos consta, pues, de:

a) unos instrumentos conceptuales: sistemas simbólicos estructurados;

b) unos modos de uso de los sistemas simbólicos: funciones cognitivas;

c) un campo de actuación: fenómenos, cuestiones y problemas. (Esquema 1).

La investigación que se presenta en este trabajo queda encuadrada, con carácter general, en esta línea de investigación y procede de la tesis doctoral Exploración de patrones numéricos mediante configuraciones puntuales, realizada por la profesora Encarnación Castro en el Programa de Doctorado de Didáctica de la Matemática de la Universidad de Granada.

\section{SISTEMAS DE REPRESENTACIÓNPARA LOS NATURALES}

\section{Sistema decimal de numeración}

El sistema decimal de numeración es una potente herramienta matemática, producto de una larga evolución histórica, inspirada por principios de economía no sólo semíbtica sino operatoria, mediante la cual el hombre ha dado cauce y expresión a sus capacidades de contar, clasificar, medir y ordenar. El dominio de este sistema es un hecho cultural básico en nuestra sociedad; su conocimiento establece un criterio para determinar que una persona ha adquirido las capacidades elementales que le permiten ocupar una posición intelectualmente digna en la sociedad. De ahí la importancia concedida en los sistema educativos a la transmisión y aprendizaje del sistema de los números naturales y de sus operaciones aritméticas elementales; el sistema decimal de numeración se utiliza como sistema de representación exclusivo.

De este modo se identifica cada uno de los números con su notación decimal y el conjunto de los naturales con la secuencia de los numerales arábigos. Tal identificación, aunque culturalmente útil, práctica y económica, es una limitación en el conocimiento de los números naturales.

\section{Análisis aritméticos}

El carácter dinámico del sistema de los números naturales queda bloqueado por la inercia de la representación decimal usual; dicho carácter dinámico establece que los números se determinan por sus relaciones mutuas. Así, conocer o saber lo que significa, por ejemplo, 15 no consiste sólo en leerlo como 1 decena y 5 unidades; también en interpretarlo como 3 veces 5,5 veces 3 , siguiente a 14 , anterior a 16 , suma de dos números consecutivos $(7+8)$, suma de tres números consecutivos $(4+5+6)$, suma de cinco números consecutivos $(1+2+3+4+5)$, anterior a un cuadrado $\left(4^{2}-1\right)$, suma de dos números por su diferencia $([4+1][4-1])$, mitad de 30, etc. Desđe esta perspectiva, cada número es un nudo en el que se entrelazan una multiplicidad de relaciones, es un elemento de una red compleja fuertemente conectada, cuyo dominio determina la comprension real que cada sujeto alcanza del sistema de los números naturales (Rico, 1995).

Las consideraciones anteriores ponen de manifiesto que, sobre la base de la notación decimal, hay otros sistemas de representación para los números naturales. Uno de ellos es el análisis aritmético de los números, considerando cada número como suma o como producto de números más sencillos. Los ejemplos anteriores son análisis aritméticos del número 15.

\section{Sistemas graficos}

No hemos tenido aún en cuenta modos de representación gráfica. La recta númerica es la representación gráfica estándar. Sobre una recta cualquiera elegimos dos puntos arbitrarios a los que asignamos los valores 0 y 1 ; por convenio, el punto que corresponde a 0 está situado a la izquierda del punto que corresponde a 1 :

\section{Figura 1}

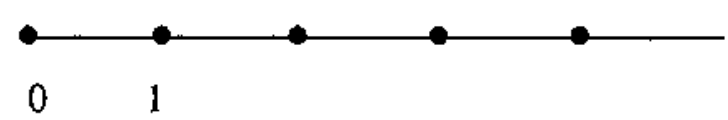


Figura 2

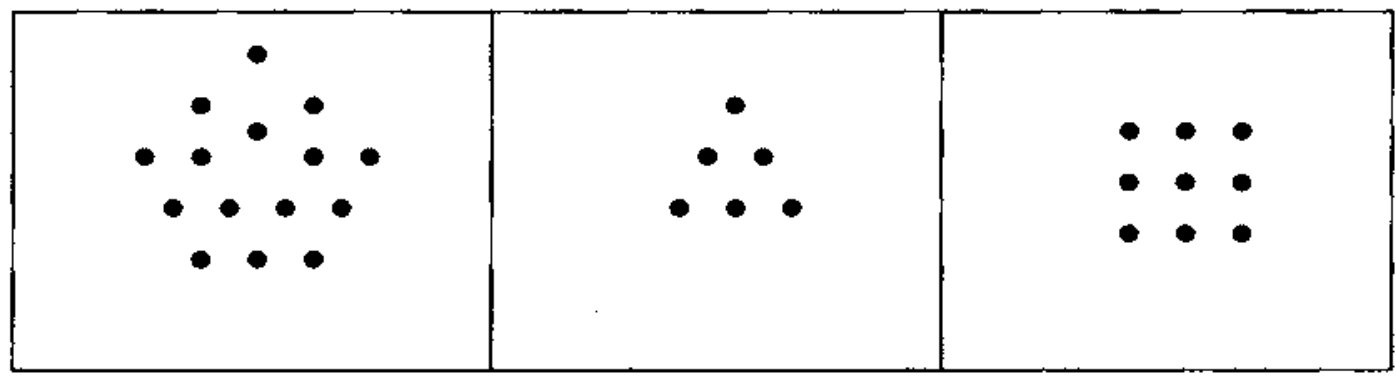

A partir de 1 y hacia la derecha, se van marcando puntos que guarden entre sî la misma distancia que los dos puntos iniciales sobre los que se van señalando, consecutivamente, los números naturales. Esta representación es un artefacto útil, cuyo empleo para el dominio del sistema de los números naturales ha sido estudiado detalladamente (Resnick, 1983)

\section{Configuraciones puntuales}

La historia nos informa de otro sistema gráfico de representación para los naturales, descuidado por el currículo escolar de matemáticas. Nos referimos a las configuraciones puntuales, utilizadas para representar números figurados y que tuvieron su origen y desarrollo en el concepto de número de la escuela pitagórica. Para los pitagóricos, el número no era simplemente una etiqueta para una colección, el símbolo de una cantidad o una construcción intelectual, sino algo que tenía consistencia en sí mismo; los números eran como una suerte de átomos que, en sus diversas composiciones y relaciones, daban la esencia misma de lo que es la variedad del mundo existente.

Eșta noción de número encontró su mejor instrumento en el sistema de representación que conocemos como configuraciones puntuales o números figurados, diferente de los sistemas de numeración al uso.
La idea básica de este sistema de representación es considerar cada número como un agregado de puntos o unidades distribuidos sobre una trama rectangular o isométrica, según una figura geométrica plana o espa* cial. De este modo aparecen números triangulares, cuadrados, rectangulares, pentagonales, piramidales y cúbicos (tantos como variantes de figuras geométricas se consideren), que facilitan pensar en cada número como un todo organizado según una estructura determinada.

\section{Precisemos:}

Las configuraciones puntuales son un sistema de representación de números, basado en un único símbolo: el punto, un espacio estructurado de representación, usualmente la trama cuadrada o la trama isométrica cuando trabajamos en el plano; un modo de organización de la cantidad de puntos que satisface criterios de simetría o regularidad convenidos y que se pueden explicitar de manera sencilla.

Este sistema de representación, al organizar geométricamente las unidades que componen cada número, propo: ciona dos informaciones importantes sobre ese número. Por un lado, visualiza al menos un análisis aritmético del número: un número triangular es una suma de números consecutivos comenzando desde 1; un cuadrado es el producto de un número por sí mismo; un rectangular es producto de dos números consecutivos. Esta informa*

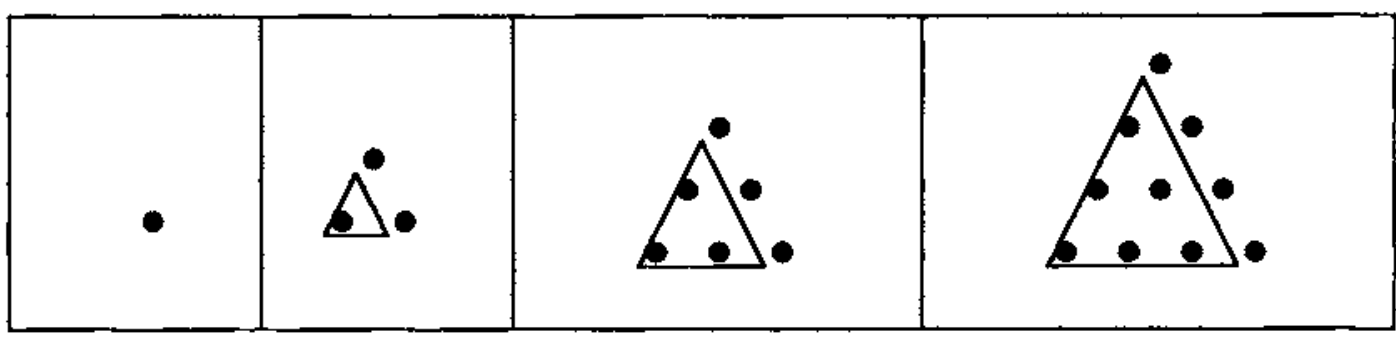


ción visual permite conocer propiedades del número en cuestión y relacionarlo con muchos otros. Además, un mismo número puede considerarse perteneciente a varios tipos de números figurados.

En segundo lugar, distintos números comparten un mismo tipo de configuración puntual. El análisis arítmético que expresá la configuración compartida se convierte en una propiedad común de los números, que puede generalizarse. De este modo el sistema de representación mediante configuraciones puntuales es un instrumento para establecer propiedades comunes a varios números y descubrir nuevas relaciones entre ellos.

Este sistema de representación permitió establecer propiedades numéricas generales e identidades algebraicas sin disponer del aparato simbólico del álgebra. El uso de números figurados lo cncontramos a lo largo de la historia de la teoría de números.

\section{HALLAZGOS EMPÍRICOS DISPONIBLES}

\section{Iniciación a las sucesiones}

El concepto de sucesión de numeros naturales es un concepto complejo, en cuya base se encuentran las nociones de conjunto totalmente ordenado con primer elemento y de proceso infinito: para todo término de la sucesión hay un siguiente. Cuando se presentan varios términos do una sucesión y se pide continuarła, la propuesta consiste en encontrar nuevos números que man. tengan unas determinadas relaciones con los términos conocidos, similares a las que éstos mantienen entre sí. Son varias las maneras mediante las que pucde relacionarse un número limitado de términos. Para la pregunta: $« 1,2,4 \ldots$ ¿cuál es el siguiente?», hay una multiplicidad de respuestas posibles. Conforme se incrementan los términos conocidos de una sucesión, las posibilidades de encontrar relaciones distintas se van Iimitando y las opciones conocidas para continuar la secuencia pueden llegar a reducirse a una sola. Reconocer las relaciones que se dan entre los términos conocidos de una secuencia permite, en ocasiones, obtener nuevos términos, es decir, continuar la secuencia. Sin embargo, la caracterización de una sucesión la da su término gencral.

\section{Términe general de una sucesión}

La expresión del término general de una secuencia de naturales presenta grandes dificultades de comprensión y es un punto al que muchos escolares son incapaces de dotar de significado adecuado por el alto grado de abstracción que supone. ¿Qué significa término general de una sucesión? El término general es la expresión algebraica de la ley que satisfacen todos los ićrminos, en función del ordinal correspondiente. El término general de una secuencia expresa la estructura común que comparten todos sus térninos cuando se les considera como elementos ordenados de un conjunto. Su modo usual de expresión es mediante notación algebrica. Así, Ia ley $a_{n}=\left(n^{2}+2 n\right) / 2$ indica que todos y cada uno de los términos de la sucesión que se considera se obtienen elevando al cuadrado el ordinal correspondiente, sumándole su doble y dividiendo el resultado entre 2 . Sin embargo, esta noción de estructura común o estructura que comparten todos los términos de la sucesión no se pone de manifiesto analizando las relaciones entre dos o tres términos consecutivos.

Disponer de varios números escritos en el sistema decimaI de numeración no permite apreciar la estructura común que tienen. Para conocer tal estructura es necesario que Jos números estén escritos mediante un desarrollo aritmético compartido o, mejor aún, que se presenten mediante una configuración puntual que se ajuste a un mismo patrón. La visualización de los números triangulares pone de manifiesto que los números $1,3,6,10,15 \ldots$ comparten un mismo patrón (Fig. 2). La traducción aritmética de ese patrón:

$$
1,1+2,1+2+3,1+2+3+4,1+2+3+4+5, \ldots
$$

adelanta Ia estructura que comparten tos números iniciales: cada uno de ellos es suma de números consecutivos, comenzando desde 1 y llegando hasta el natural que corresponde a su posición ordinal en la secuencia. Pero aún quedan muchos fenómenos de comprensión que considerar para establecer que la ley de esa sucesión es, precisamente:

$$
a_{n}=\left(n^{2}+2 n\right) / 2
$$

\section{Contextualización curricular}

El trabajo Exploración de patrones numéricos mediante configuraciones puntuales (Castro, 1994) se propone estudiar la viabilidad, en el currículo de matcmáticas de secundaria obligatoria, de un sistema de representación para los números naturales que proporcione un instrumento de visualización y análisis para las sucesiones análogo a la representación gráfica de las funciones. Estudiamos la potencia de las configuraciones puntuales para expresar relaciones y propiedades numéricas; también estudiamos como, mediante dichas representaciones, los estudiantes descubren y utilizan las propiedades numéricas.

Este estudio se organiza basándose en las referencias siguientes:

- empleo coordinado de los tres sistemas de representación mencionados para números naturales: configuraciones puntuales, sistema decimal de numeración y desarrollo aritmético de los números;

- trabajo con secuencias numéricas, lineales y cuadráticas, analizando el patrón que definen cada una de ellas mediante las configuraciones puntuales y los desarrollos operatorios;

- realización de las siguientes tareas: continuar una secuencia, extrapolar términos, generalizar, obtener el término general y utilizar el término general para obtener términos concretos. 


\section{DISCUSIÓN DE HALLAZGOS}

\section{Sucesiones y sistemas de representación}

Las configuraciones puntuales permiten representar los términos de las sucesiones de números naturales de primer o segundo grado. Las progresiones aritméticas admiten representaciones puntuales sencillas, por to general rectangulares de base o altura constantes, o bien variantes de esta representación. Se denominan secuencias lineales porque la representación gráfica de sus términos puede analizarse mediante una descomposición en líneas y la diferencia entre dos términos se puede describir como agregación de una línea.

Figura 4

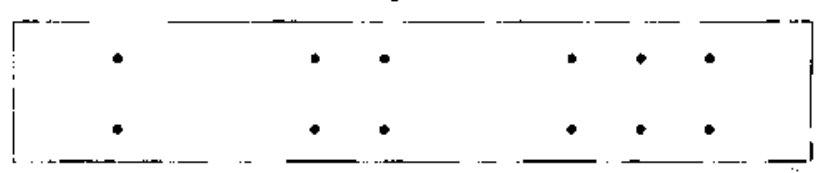

Las sucesiones de diferencias segundas constantes son aquéllas cuyo término general viene dado por un polinomio de segundo grado. Los casos más sencillos son la sucesión de números cuadrados, $C_{n}=n^{2}$, y la de números rectangulares, $R_{n}=n(n+l)$.

La representación gráfica de los términos de estas sucesiones se puede realizar teniendo en cuenta que las dos dimensiones son variables; el paso de un término al siguiente viene dado por un aumento de superficie según las dos dimensiones. La estructura de estos números se llama cuadrática o multiplicativa y el paso de un término al siguiente no es constante sino que, al aumentar en dos dimensiones, también es variable, siendo esa variación lineal.

Figura 5

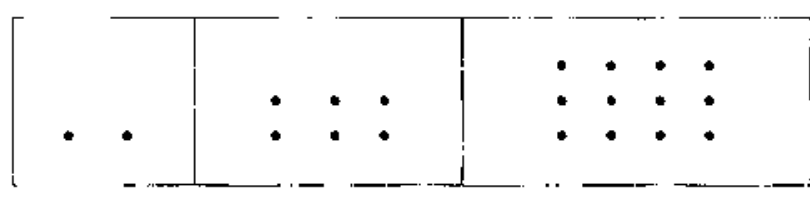

En general, si la ley de una sucesión es $a n=a n^{2}+b n+c$, dicha sucesión es de diferencias segundas constantes. Si $a_{n}$ toma valores naturates para todo $n$, entonces cada término puede representarse mediante una configuración plana poligonal, regular o irregular, manteniendo todos los términos un mismo patrón de representación.

\section{Diversidad de desarrollos}

Hemos introducido a los escolares del primer ciclo de secundaria (12-14 años) en el sistema simbólico de representación de las configuraciones puntuales. Hemos utilizado estas representaciones como sistema simbólico alternativo con el que realizar, entre otras, Las siguientes tareas: visualizar el patrón de representación que comparten los términos de la sucesión, continuar la secuencia y representar términos avanzados con este patrón. Así, en el ejemplo de la figura 4, los alumnos reconocen la forma geométrica de los tres términos representados, añaden dos o tres términos a continuación y también son capaces de representar el término $11^{\circ}$ o el $15^{\circ}$.

Igualmente, la representación puntual facilita el análisis estructural de los términos de la sucesión y permite expresar nuevos términos mediante el desarrollo aritmético obtenido. Para el ejemplo de la figura 4 son varios los desarrollos aritméticos correctos encontrados por los alumnos:

a) $2,3+3,4+4+4,5+5+5+5, \ldots$

b) $1+1,2+2+2,3+3+3+3,4+4+4+4+4, \ldots$

c) $2 \times 1,3 \times 2,4 \times 3,5 \times 4, \ldots$

d) $1^{2}+1,2^{2}+2,3^{2}+3,4^{2}+4, \ldots$

e) $2^{2}-2,3^{2}-3,4^{2}-4,5^{2}-5, \ldots$

Se observa que las posibilidades de análisis del patrón puntual son variadas; cada una de ellas proporciona un posible desarrollo aritmético (aditivo o multiplicativo) que comparten los términos de la secuencia. Se obtienen asi diversas expresiones de los términos de la sucesión considerada, en el sistema de representación que hemos denominado análisis aritmético. Cuando esta misma sucesión se presenta escrita en el sistema decimal de numeración: $2,6,12,20 \ldots$, los alumnos no disponen de datos suficientes para encontrar un desarrollo aritmético común a estos términos.

A partir de las conexiones establecidas entre los términos de la secuencia de configuraciones puntuales y la secuencia de desarrollos aritméticos, hemos estudiado la comprensión que muestran los escolares para generatizar la estructura común que tienen los términos de una secuencia. Es decir, tratamos de explicitar la noción de término general de una sucesión que tienen los escolares de 13-14 años mediante la pregunta: ¿Cómo se escribe el têrmino que ocupa el lugar n-ésimo? Las respuestas a esta cuestión varían según el sistema de representación considerado.

Así, en el sistema decimal de numeración, el término general de la sucesión viene dado por $n$, que es la traducción simbólica inmediata de las expresiones: «un número en general», «cualquier número de la sucesión», «cualquier término de la sucesión»... o equivalentes.

Al emplear el patrón geométrico, la necesidad de dejar espacios vacíos entre los puntos para indicar el paso a $n$ lieva a que algunos alumnos utilicen modelos continuos para el término general. Esto indica una dificultad de este sistema para las expresiones generales. 
En la representación por desarrollos aritméticos encontramos que el paso al término general se suele escribir adecuadamente. Sin embargo, cuando se dispone de varias expresiones que traducen el mismo patrón, no es fácil considerar equivalentes el paso de cada una al término general.

Se detecta de este modo un fuerte obstáculo para la obtención de la expresión del término general de una sucesion. Tanto las representaciones puntuales como los desarrollos aritméticos expresan, de algún modo, la estructura que comparten varios números. La notación decimal de esos mismos números no pone de manifiesto la estructura común.

Cuando se pide obtener el término general de la sucesión, lo que se pide es encontrar, mediante notación algebraica, una expresión general de la estructura común de todos los términos. Esta pregunta no se puede responder en el sistema decimal de numeración, ya que, en este sistema, cada término viene dado por un símbolo único y no se considera su estructura compartida; de ahí que la respuesta más común que se encuentra es $n$, que es un símbolo único y representa «un término en general». En la representación mediante configuraciones puntuales sí se aprecia la estructura común, pero el carácter concreto de tales representaciones dificulta la obtención de un término genérico. S6lo mediante los desarrollos aritméticos es posible generalizar las expresiones de los términos de una sucesión.

\section{CONCLUSIÓN}

Nuestro trabajo se ha centrado en el estudio de las sucesiones de números naturales, lineales y cuadráticas, mediante el empleo de los tres sistemas de representación descritos: figurativo (configuraciones puntuales), simbólico estructurado (sistema decimal de numeración) y operatorio (desarrollos aritméticos). De este modo resulta posible hacer hincapié en los patrones de formacion de las secuencias, tanto puntuales como numéricas.

Los resuitados obtenidos en este estudio han puesto de manifiesto que los escolares admiten sin dificultad el sistema de representación puntual para los números y lo utilizan adecuadamente, trabajando con diferentes modelos geométricos; enuncian una gran riqueza de relaciones para números triangulares y cuadrados; y establecen argumentos que conectan el patrón geométrico y su correspondiente patrón aritmético a través de las representaciones puntuales.

Los trabajos realizados por los alumnos a partir de las tareas propuestas han mostrado que, de los tres sistemas empleados en la representación de números, la configuración puntual es el más intuitivo debido a stu carácter gráfico, lo que permite un tratamiento y análisis visual de la estructura de una cantidad. Sin embargo, este sistema adquiere su mayor potencia cuando se trabaja conjuntamente con los desarrollos aritméticos y la notación decimal usual. Una configuración puntual adquiere sentido cuando se emplea como visualización de un determinado desarrollo aritmético de un número -o familia de números - concretos. La variedad de desarrollos que puede sugerir una misma representación puntual pone de relieve este carácter intuitivo.

Desde el punto de vista aritmético, la incorporación del sistema de representación denominado configuración puntual proporciona un instrumento para analizar los números y obtener diferentes desarrollos aritméticos de un mismo número. El carácter operatorio que proporciona el nuevo sistema simbólico a los números naturales queda de manifiesto durante el trabajo realizado. Se consigue de este modo una diversidad de desarrollos aritméticos para cada número. También se refuerza la idea de que hay números que comparten estructura aritmética; dicha estructura se visualiza mediante un patrón geométrico y se expresa mediante un desarrollo aritmético. Esta noción es un primer paso hacia la generalización sobre base aritmética.

La riqueza de relaciones entre números que comparten patrón es un tercer dominio de conocimientos que los alumnos ponen de manifiesto y en el que aparecen los primeros intentos de generalización en expresiones numéricas.

Hemos comprobado que es muy débil la integración entre los tres sistemas simbólicos para expresar la noción de término general de una sucesión. Son muy pocos los alumnos que identifican el término general con la estructura operatoria común que comparten los términos de una secuencia, cuya notación más adecuada viene dada mediante el đesarrollo aritmético. La comprensión de los escolares de 12-14 años sobre la noción de término general es prácticamente inexistente dado que no se aprecia estructuración entre las representaciones mentales correspondendientes a los diferentes sistemas de representación utilizados. Sólo unos pocos alumnos, que integran total o parcialmente los tres sistemas, muestran un cierto dominio de esta noción, si bien se aprecian grados distintos de comprensión.

Hemos obtenido evidencias que permiten sostener nuestra tesis principal: la riqueza y complejidad de las estructuras numéricas necesitan de varios sistemas de representacion complementarios; la aportación de las representaciones gráficas es esencial para comprender determinadas nociones estructurales.

Es necesaria la integración entre varios sistemas de representación para poner de manifiesto las dificultades de conceptos como el de término general de una sucesión y para establecer vías que permitan la superación de tales dificuitades mediante la comprensión de las estructuras subyacentes. 


\section{REFERENCIAS BIBLIOGRÁFICAS}

BADIOU, A. (1990). Le nombre et les nombres. París: Editions du Seuil.

BEHR, M., LESH, R., POST y SILVER, E. (1983). Rational Number Concepts, Lesh, R. y Landat, M. (eds.). Acquisition of Mathematics concepts and processes. Nueva York: Academic Press.

BEILER, A. (1966). Recreations in the theory of numbers. Nueva York: Dover.

CARPENTER, T., FENNEMA, E. Y ROMBERG, T. (1993). Rational Numbers. An Integration of Research. Hillsdale: Lawrence Erlbaum Associates.

CASTRO, E. (1994). Exploración de patrones numéricos mediante configuraciones puntuales. Estudio con escolares de primer ciclo de secundaria (12-14 años). Tesis doctoral. Granada: Universidad de Granada.

DANCING, J. y SOSA, E. (1993). A Companion to Epistemology. Oxford: Basil Blakcwell.

DUVAL, R. (1993). Semiosis et Noesis, Lecturas en Didáctica de la Matemática: Escuela Francesa. México: Sección de Matemática Educativa del CINVESTAV-IPN.

DUVAL, R. (1995). Semiosis et Pensée humaine. Berna: Peter Lang, SA.

FEFERMAN, S. (1989). The Number Systems. Foundations of Algebra and Analysis. Nueva York: Chelsea Publishing Company.

FEY, J. (1990). Quantity, en Steen, L. (ed.). On the shoulders of giants. New approaches to numeracy. Washington: National Academy Press.

FERRATER, J. (1981). Diccionario defilosofía. Madrid: Alianza Editorial.

GOLDING, G. (1993). The IGPME Working Group on Representations. Proceedings of the Seventeenth International Conference for the Psychology of Mathematics Education. Tsukuba: Universidad deTsukuva.

GONZALEZ, J.L. (1995). El campo conceptual de los números naturales relativos. Tesis doctoral. Granada: Universidad de Granada.

GUTTENPLAN, S. (1994). A Companion to the Philosophy of Mind. Oxford: Blackwell

HIEBERT, J. y CARPENTER, T. (1992). Learning and teaching with understanding, en Grouws, D.A. (ed.). Handbook of Research on Mathematics Teaching and Learning. Nueva York: MacMillan Publishing Company.
JANVIER, C. (1978). The interpretation of complex cartesian graph representing situations-studies and teaching experiments. Tesis doctoral. Universidad de Nottingham.

IANVIER, C. (ed.). (1987). Problems of representations in the teaching and learning of mathematics. Londres: Lawrence Earlbaum Associated, publishers.

KAPUT, J. (1987). Representation systems and Mathematics, en Janvier, C. (ed). Problems of representation in the teaching and learning of mathematics. Hillsdale: LEA.

KAPUT, J. (1992). Technology and Mathematics Edtication, en Handbook of Research on Mathematics Teaching and Learning. Grouws, D.A. (ed.). Nueva York: MacMillan Publishing Company.

KIERAN, C. y FILLOY, E. (1989). El aprendizaje del álgebra escolar desde una perspectiva psicológica. Enseñanza de las Ciencias, Vol. $7(3)$.

KITCHER, P. (1984). The Nature of mathematical knowledge. Oxford: Oxford University Press.

KÖRNER, S. (1977). Introducción a la filosofía matemática. México: Siglo XXI.

RESNICK, L. (1983). A developmental theory of number understanding, en Ginsburg, H. P. (ed.). The development of mathematical thinking. Orlando: Academic Press.

RICO, L. (1995). Conocimiento numérico y formación del profesorado. Granada: Universidad de Granada.

ROMERO, I. (1995). La introducción del número real en educación secundaria. Tesis doctoral. Granada: Universidad de Granada.

SHELL CENTRE (1986). The language of functions and graphs: An examination module for secondary school. Manchester: Joint Matriculation Board.

SLOANE, N. (1973). A handbook of integer sequences. San Diego, California: Academic Press

VON NEUMANN, J. (1983). The Formalist Foundation of Mathematics, en Benacerraf,P. y Putnam, H.(eos.). Philosophy of mathematics. Selected readings. Cambridge: Cambridge University Press

WIGGENSTEIN, L. (1988). Investigaciones filosóficas. Barcejona: Crítica.

WITTROCK, M. (1990). La investigación de la enseñanza. III. Profesores y alumnos. Madrid: Paidós Educador. 
